\title{
Hacia otra dimensión juridica: el derecho de los robots*
}

\section{Ana Karin Chávez Valdivia**}

\begin{abstract}
RESUMEN
Un nuevo escenario en el cual el creciente y rápido desarrollo e interés por introducir robots artificialmente inteligentes en la vida cotidiana de los seres humanos hace necesario replantear la capacidad de nuestros sistemas legales actuales para manejar los casos que involucran a estos actores.

Durante mucho tiempo la fabricación y el diseño de robots han enfrentado la posibilidad de demandas por responsabilidad del producto. Ahora, sin embargo, se desarrollan robots sociales que están diseñados para interactuar con los humanos de forma intima cuando estamos en la forma más humana y vulnerable. Con este cambio de propósito, la robótica se está aventurando en ámbitos legales que tradicionalmente han gobernado las interacciones sociales entre humanos, en lugar de las interacciones entre humanos y máquinas.

Asistimos a una nueva generación de sistemas robóticos como consecuencia de la evolución tecnológica lo que significa que en el plano jurídico empiezan a quedar atrás los debates y cuestiones de carácter general sobre las telecomunicaciones e internet para ahora cobrar especial importancia la regulación de las tecnologias emergentes y disruptivas.

Consideramos que la regulación jurídica de los robots deviene en un tema de obligado análisis interdisciplinario. Más aún cuando enfrentamos la posibilidad de que una nueva categoría de sujeto jurídico -que se encontraría a medio camino entra la persona y el objeto- nos conducirá, en algún momento, a abordar la sustantividad y viabilidad de un Derecho de los Robots.
\end{abstract}

\section{PALABRAS CLAVE}

Inteligencia artificial, robótica, personalidad electróni$c a$, derecho de los robots

\begin{abstract}
Anewscene where the increasing and quick development and interest in insert intelligent synthetically robots in human being daily life makes necessary evaluate the capacity of our current legal system in order to handle cases where these actors are involved.

For long time ago robots manufacturing and drawing have dealt with the possibility of claims for product responsibility. However at the present social robots are being carried out to interact with humans in an intimate way in those moments and places we are in our most human and vulnerable face. With this change of purpose, robotics is venturing into legal fields which traditionally have ruled social interactions among humans, instead of interactions between humans and machines.

We are attending new generation of robotic systems as a result of technological development what means that in legal level discussion and matters of general character on telecommunications and internet are lagging behind and emergent and disruptive technologies regulation are increasing in importance. We consider that robot legal regulation evolves into an obligatory subject of interdisciplinary analysis. Furthermore facing the possibility of a new category of legal person - placed in halfway of the person and the object-will lead us to tackle the substantivity and viability of a Law of Robots at some point.
\end{abstract}

\section{KEY WORDS}

Artificial intelligence, robotics, electronic personality, law of robots

\footnotetext{
*Artículo de Investigación postulado el 4 de febrero de 2020 y aceptado el 27 de julio de 2020

${ }^{*}$ Docente investigadora en la Universidad La Salle, Arequipa, Perú. (achavez@ulasalle.edu.pe) orcid.org/0000-00026453-3119
} 


\section{SUMARIO}

1. Introducción

2. Consideraciones generales

3. Robótica e Inteligencia artificial

4. Hacia una personalidad de los robots

5. Sobre la viabilidad de un Derecho de los Robots

6. Consideraciones finales

7. Referencias

\section{Introducción}

La idea de que los robots hayan evolucionado más allá de la consideración como "propiedad" y la viabilidad de que adquieran estatus legal como seres con "derechos" plantea múltiples interrogantes sobre diversos temas.

La base axiológica de los ordenamientos jurídicos occidentales se centra en la dignidad inherente de la persona humana. Este hecho implica entre otras cosas el "derecho a tener derechos". Los sistemas legales se basan en un conjunto de suposiciones filosóficas en el que los seres humanos ocupan una posición excepcional; esto resulta no solo del hecho de que las normas legales son establecidas por ellos, sino también, o más aún, del valor moral inherente del ser humano como un fin en sí mismo. En este sentido el derecho se basa en el supuesto de que todas las relaciones jurídicas tienen lugar entre las personas naturales y/o jurídicas, es decir los seres humanos y sus entidades orgánicas.

La creciente e imparable introducción de la inteligencia artificial en la industria y en la sociedad promete revolucionar las estructuras sociales actuales y comportará no solo innumerables desafíos regulatorios que los actuales marcos legales -en su mayoría- no están preparados para enfrentar; sino también tecnológicos e industriales.

La evolución de la inteligencia artificial y la consecuente inserción de nuevos agentes inteligentes en la sociedad y en la vida cotidiana que comenzarán a operar sin la intervención de humanos y eventualmente incluso sin su conocimiento, marcarán un hito en el desarrollo del derecho; situándolo en la necesidad de replantearse un nuevo tipo de persona no concebida por los hasta ahora ordenamientos jurídicos: la "persona electro-física". 


\section{Consideraciones generales}

Si nos preguntamos porque la inteligencia artificial (IA) genera tanta polémica, probablemente las primeras respuestas se orientarán hacia cuestiones intelectuales compuestas de temores fundamentados en amenazas a la subsistencia humana en sus diversas formas.

El tema de la regulación de los robots lleva varios años en la mira de diversos especialistas e instituciones públicas. En este sentido "Los derechos legales de los robots" es un tema que se ha debatido por más de 30 años, comenzando con un artículo de Robert Freitas Jr. en $1985 .{ }^{1}$

El tiempo ha demostrado que el debate teórico debe evolucionar en leyes y que tal vez éstas trastocarán los ordenamientos jurídicos como han sido hasta ahora conocidos.

En un contexto jurídico en el que las normas existentes establecen que entidades como empresas, corporaciones, asociaciones $u$ otras son tratadas como cierto estatus legal; el desarrollo tecnológico demanda centrar nuestra atención en la posibilidad de que pueda ocurrir lo mismo con los robots inteligentes autónomos.

En los últimos años el tema de la personalidad del robot ha pasado a un primer plano en la robótica social gracias a diversas publicaciones que abordan este planteamiento desde diversas aristas. ${ }^{2}$ Aceptar la posibilidad de que los robots estén dotados de algún tipo de personalidad artificial implica un proceso de transición que no será el mismo en todos los países ni contará con el mismo nivel de aceptación debido a innumerables factores, pero que aun siendo así, las sociedades deberán afrontar en un corto periodo de tiempo.

\footnotetext{
${ }^{1}$ Freitas Jr., R.: "The legal rights of robots". Student Lawyer 13, pp. 54-56 (1985)

${ }^{2}$ Entre los títulos más resaltantes podemos mencionar: Steffen Wettig y Eberhard Zehendner a través del artículo "The electronic agent: A legal personality under German law" (2003). Samir Chopra y Laurence F. White en su libro titulado: "A Legal Theory for Autonomous Artificial Agents" (2011). F. Patrick Hubbard a través de su artículo: Do androids dream?: Personhood and intelligent artifacts publicado en la revista Temple Law Review (2010). Patrick Lin; Keith Abney y George A. Bekey mediante el artículo denominado "Can machines be people? Reflections on the turing triage test" publicado en el libro Robot Ethics: The Ethical and Social Implications of Robotics (2012). Kamil Muzyka mediante su escrito titulado: "The outline of personhood law regarding artificial intelligences and emulated human entities" publicado en Journal of Artificial General Intelligence (2014). Evan Joseph Zimmerman a través de su artículo denominado: "Machine Minds: Frontiers in Legal Personhood" publicado en la revista de Derecho de la Universidad de Berkeley (2015).
} 


\section{Robótica e Inteligencia artificial}

Es indiscutible que los avances en robótica tienen cada vez mayor aplicación y difusión en la sociedad por lo que su integración como parte del entorno no se vislumbra tan inviable. Gobiernos, universidades y el sector privado han mostrado un interés creciente en torno al tema. Así tenemos a Google que ha adquirido varias empresas de inteligencia artificial; y Amazon que en el año 2012 compró una empresa de robótica para ayudar a automatizar sus almacenes ${ }^{3}$ y a finales del 2014 anunció un proyecto para entregar algunos paquetes empleando drones. ${ }^{4}$

La robótica como la siguiente tecnología transformadora de los ordenadores e internet, desde hace algunos años ya es una realidad y viene generando diversas cuestiones jurídicas y políticas que en la mayoría de casos desborda el marco del Derecho Informático, llevándonos a cuestionarnos sobre la viabilidad de un Derecho de los Robots.

No existe, sin embargo, un consenso sobre el concepto de robot ya que depende en gran medida del lugar y cultura donde se concibe siendo además un concepto variable en el tiempo. A esto se suma que muchas disciplinas científicas y tecnológicas están directamente relacionadas con diferentes tipos de robot y que en la industria la diversidad es bastante amplia. Por estas razones esbozar una clasificación deviene en una tarea bastante compleja. Sin embargo compartimos el criterio de Barrientos, Peñin, Balaguer y Santoja quienes distinguen cuatro tipos de robots y tres generaciones de desarrollo en función a su complejidad y adaptabilidad de interacción con el entorno. ${ }^{5}$

En nuestro artículo titulado: "Hacia el quebrantamiento de paradigmas jurídicos: La robótica y la inteligencia artificial, abordamos esta nueva forma de "singularidad" con potencialidad para convertirse en "agente moral" tras el análisis de diversas consideraciones. ${ }^{6}$ En este sentido la esencia de un robot es que la tecnología combine y se construya sobre el paradigma de: sentir- pensar

\footnotetext{
${ }^{3}$ Pooler, Michael, "Los robots de Amazon revolucionan la logística". Expansión. [Consulta: 4 de marzo de 2019]. Disponible en: http://www.expansion.com/economia-digital/innovacion/2017/08/29/59a458d7ca4741cf138b4622.html ${ }^{4}$ Castromil, Juan, "Amazon usará drones Prime Air para repartir sus paquetes volando." CLIPSET. [Consulta: 2 de marzo de 2019]. Disponible en: https://clipset.20minutos.es/amazon-usara-drones-prime-air-para-repartir-sus-paquetes/ ${ }^{5}$ Barrientos, Antonio, Peñin, Luis, Balaguer, Carlos y Aracil, Santoja, Fundamentos de la Robótica, Madrid, Editorial Mc Graw Hill, 2007, pp. 10-11.

6 Artículo presentado en el XXI Congreso Iberoamericano de Asociaciones de Derecho e Informática. San Luis de Potosí - México. 2017
} 
-actuar tal como sostienen Singer ${ }^{7}$ o Calo $^{8}$ en los Estados Unidos y Palmerini ${ }^{9}$ en Europa. Es decir, deben contar con tres componentes:

- Sensores que vigilan el entorno y detectan cambios en él.

- Procesadores o inteligencia artificial que deciden como responder.

- Actuadores que operan sobre el entorno de manera que refleje las decisiones anteriores, provocando algún tipo de cambio en el entorno mundo de un robot.

Cuando estos tres factores actúan conjuntamente podemos decir que nos encontramos ante un robot que adquiere funcionalidad de un organismo artificial capaz de operar independientemente, libre de la intervención humana (u otra) y por extensión, libre de condicionantes externos.

Sin embargo como acertadamente señala Barrio, al igual que los elementos fundantes de Internet interactúan con el Derecho en formas novedosas y originan problemas jurídicos insospechados, así también las características esenciales de la robótica están produciendo situaciones jurídicas disruptivas. ${ }^{10}$

En esta línea de pensamiento, Calo propone una sistematización que genera diversos cuestionamientos doctrinarios al señalar que la robótica entraña tres propiedades privativas (o transformativas):

a) Corporeidad. Frente al software, el robot es material o con una materialidad corpórea.

b) Impredecibilidad. A diferencia de una simple máquina, el robot piensa y decide con cierta autonomía.

c) Impacto social; que en determinados androides lleva a las personas a preocuparse por su situación, o incluso, hasta por sus "derechos".11

\footnotetext{
${ }^{7}$ Singer, Peter, Wired for War: the robotics revolution and confliction in the 21st century, New York, Editorial Penguin Press, 2009, pp. 37.

${ }^{8}$ Calo, Ryan, "Robots and ethics", en Lin, Patrick (coord.), Robot ethics: The ethical and social implications of robotics., Cambridge, MIT Press, 2012, pp. 159-168.

${ }^{9}$ Palmerini, Erica y Bertolini, Andrea, "Liability and risk management in robotics", en Schulze, Reiner., y Staudenmayer, Dirk (coords.), Digital Revolution: Challenges for Contract Law in Practice, Baden-Baden, Nomos, 2016, pp. 225-260.

${ }^{10}$ Barrio, Moises, "Del derecho de internet al derecho de los robots", en Barrio, Moises (coord.), Derecho de los Robots, Madrid, Wolters Kluwer, 2018, pp. 61-86.

${ }^{1 "}$ Calo, Ryan, "Robots as legal metaphors", Harvard Journal of law and Technology, Estados Unidos, No. 30, 2016, pp. 209-237.
} 
En cuanto a este último punto el autor expresamente alude a una "nueva categoría de sujeto jurídico a medio camino entre persona y objeto". Señalando que la calificación "a mitad de camino"; es parcial, porque la asignación de estatus puede ser incompleta, contextual, inestable y sobre todo oportunista en el sentido que los ciudadanos pueden tratar al robot como "persona" para algunos propósitos u como "objeto" para otros.

Compartimos la opinión de Filipe Maia Alexandre al señalar que, si bien en teoría la robótica y la inteligencia artificial pueden permitirle a la humanidad completar su búsqueda definitiva de bienestar social y prosperidad, el camino que actualmente nos separa de ella es largo y borroso. Como cualquier otra gran revolución en la industria, ésta también tendrá varios desafíos que deberá superar para poder llevarse a cabo con éxito. Señala además que con la creación de la inteligencia artificial, la humanidad está a punto de entrar en un territorio inexplorado y transitar un camino que nunca antes se había tratado. ${ }^{12}$

Conceptualizar la inteligencia artificial (IA), desde hace años no reviste tanta simplicidad, debido fundamentalmente a dos razones: En primer lugar porque no hay consenso sobre de que se trata la inteligencia propiamente dicha y en segundo lugar, en consideración de algunos sectores, no existen muchas razones para creer que la inteligencia de una máquina tenga gran relación con la inteligencia humana.

Si bien es cierto que han existido y existen muchas propuestas de definición con diversos enfoques, la mayor parte de ellas -en términos muy generales- se han centrado en la idea de crear programas de ordenador o máquinas capaces de tener conductas que se pueden considerar inteligentes si fuesen realizadas por un humano. Ya en 1955 John McCarthy, uno de los fundadores de la IA junto a Minsky, Rochester y Shannon describieron el proceso como: "El hacer que una máquina se comporte de formas que serían llamadas inteligentes si un ser humano hiciera eso".

En este sentido, tal como señala García-Prieto; disciplinas del control inteligente, el control multiagente, los sistemas expertos o la programación semántica que tradicionalmente han sido muy influyentes en la robótica, también han jugado un papel fundamental en el desarrollo de la IA que de manera circular vuelve a retroalimentar a la robótica. Otras disciplinas, además, relacionadas con la teoría informática, como el soft computing, la lógica difusa, la neurocomputación, la computación evolutiva, la computación distribuida e incluso la computación caótica; han tenido influencia considerable en la robótica y en

\footnotetext{
${ }^{12}$ Maia, Filipe, The Legal Status of Artificially Intelligent Robots, Tilburg, Universidad de Tilburg, 2017, pp. 6.
} 
la IA. Finalmente, señala el autor que respecto a las matemáticas y mus especialmente la estadística, las redes neuronales artificiales, las redes bayesianas, el machine learning y el pattern recognition son básicamente las principales disciplinas que conforman lo que se entiende hoy habitualmente como IA ${ }^{13}$. En suma, resulta muy difícil establecer los límites de lo que es - y lo que deja de ser- la inteligencia artificial.

Sin embargo, García- Prieto refiere también que si es posible comprender que esta técnica posee una cierta ontología diferenciada. Si bien la terminología utilizada depende bastante del ámbito en el que se trabaje, existe un esquema básico que se puede generalizar a lo largo de cualquier desarrollo de IA, siendo así y en base a esta consideración el autor la conceptualiza como "toda técnica de procesamiento de información caracterizada por hacer cálculos sobre determinada información en un espacio dimensional virtual y construido mediante operaciones -generalmente no lineales- llevadas a cabo dentro del propio algoritmo para aprovechar diversas propiedades de espacios altamente dimensionales". Precisando que, suele clasificar a estas técnicas una forma de trabajo estadístico orientado a la clasificación de patrones a partir de ejemplos, aunque esta característica es más bien metodológica.

En este orden de ideas, debe tenerse presente que para comprender la influencia real que la IA ha tenido en la robótica es necesario conocer -al menos superficialmente- como funcionan gran parte de las metodologías. Cabe mencionar sin embargo, que al no ser ese el objeto del presente artículo éstas no serán abordadas.

Consideramos que conforme avance la innovación tecnológica dejará de existir una distinción práctica entre los robots y los agentes de inteligencia artificial y se unirán en una categoría única. Al mismo tiempo debe tenerse presente que tanto los robots en cuanto entidades dotadas de materialización física pero también de un sistema de software presentan la potencialidad y riesgos del mundo físico y del virtual.

\section{Hacia una personalidad de los robots}

El Derecho parte de la distinción básica entre personas y cosas. Los defensores de los robots advierten que cuanto más antropomórfico éstos sean, más

\footnotetext{
${ }^{13}$ García-Prieto, Juan, "¿Qué es un robot?", en Barrio, Moisés (coord.), Derecho de los Robots, Madrid, Wolters Kluwer, 2018, pp. 25-60.
} 
personas tienden a compartir la culpa con el robot por el fracaso y el elogio por el éxito.

En este sentido Darling analiza si la forma en que las personas parecen reaccionar frente a las máquinas antropomorfas sugiere la necesidad de extender un conjunto limitado de derechos legales a los robots sociales o, al menos prohibiciones contra su abuso, aun cuando no sean reputados como seres vivos o sensibles a un nivel racional. ${ }^{14}$

Por otro lado, a juicio de la opinión dominante, sólo del hombre pude decirse -según señala Hermogeniano en el Digesto- que en razón suya está constituido todo el derecho. No obstante, no podemos negar que muchos países ya están asistiendo al debate acerca de crear o no una nueva categoría de sujeto jurídico. Tal es así que el Parlamento de la Unión Europea ${ }^{15}$ en su Resolución formula una serie de consideraciones precisas, así como recomendaciones a efecto de regular a corto plazo la creación, uso y efecto de la robótica.

En este contexto debe hacerse mención a la posible creación de una personalidad jurídica específica ${ }^{16}$ es decir la creación de una "personalidad electrónica” para aquellos supuestos en los que los robots tomen decisiones autónomas inteligentes. Cabe señalar al mismo tiempo, que el concepto de personalidad jurídica en sí mismo no ha sido una realidad inmutable a lo largo de la historia, basta con hacer un recorrido remontándonos a sus orígenes en el siglo XIII hasta el año pasado con el estado del Whanganui. ${ }^{17}$

Por otro lado; como acertadamente sostiene Barrio Andrés, la personalidad electrónica puede ser reputada como un enfoque plausible al problema de la responsabilidad, tanto para $\operatorname{los}^{18}$ robots dotados de un cuerpo como para los robots software que exhiben un cierto grado de autonomía e interactúan con

\footnotetext{
${ }^{14}$ Darling, Kate, "Extending legal protection to social robots: The effects of anthropomorphism, empathy, and violent behavior towards robotic objects", en Calo, Ryan (coord.), Robot Law, Reino Unido, Edward Elgar, 2012, pp. 23.

${ }^{15}$ Resolución del Parlamento Europeo Resolución del Parlamento Europeo, de 16 de febrero de 2017, con recomendaciones destinadas a la Comisión sobre normas de Derecho civil sobre robótica (2015/2103(INL))

${ }^{16}$ INFORME con recomendaciones destinadas a la Comisión sobre normas de Derecho civil sobre robótica (2015/2103(INL)) Comisión de Asuntos Jurídicos, señala en la sección sobre responsabilidad: "Crear a largo plazo una personalidad jurídica específica para los robots, de forma que como mínimo los robots autónomos más complejos puedan ser considerados personas electrónicas responsables de reparar los daños que puedan causar, y posiblemente aplicar la personalidad electrónica a aquellos supuestos en los que los robots tomen decisiones autónomas inteligentes o interactúen con terceros de forma independiente".

${ }^{17}$ El acuerdo T tohu Whakatupua es el nombre del contrato con que se marca la obligación de las partes de respetar el tratamiento que el pueblo Ivos tiene al río Wanganui, situado en la Isla del Norte de Nueva Zelanda, firmado entre Nueva Zelanda y la gente local Whanganui Ivi el 30 de agosto de 2012, proceso iniciado en el año 1994. El acuerdo en mención reconoce la existencia de una nueva entidad legal - Te Awa Tupua. El Awa Tupua es la "persona jurídica" del río Whanganui. 
las personas. Siendo así, las propiedades señaladas anteriormente resultarán relevantes en los diversos contextos jurídicos como son el Derecho Penal y procesal, la Responsabilidad civil, la Propiedad intelectual, Libertad de expresión, privacidad, Derecho de los contratos, Derecho tributario, entre otros.

En consecuencia; las cualidades esenciales o distintivas de los robots y sus indiscutibles repercusiones en el campo del derecho nos conduce a plantearnos la viabilidad de una rama propia: El Derecho de los Robots. Este hecho no debe sorprendernos dado que en su momento la irrupción de Internet en nuestras vidas, su desarrollo y evolución; así como las implicancias jurídicas que trajo consigo, significó que el Derecho tuviera que replantearse infinidad de temas jurídicos siendo resultado de ese proceso el nacimiento de una nueva rama del derecho conocido mayoritariamente en nuestro país como Derecho Informático; rama que recibe otros nombres en diferentes países pero cuyo objeto de estudio es sustancialmente el mismo.

Si bien somos conscientes que este planteamiento -que dividió a Europa en su momento al punto que expertos de más de catorce países mostraron su rechazo en torno a la posibilidad de otorgar "personalidad" a los robots alegando; entre diversos argumentos, una sobrevaloración a sus capacidades reales, un imprevisible impacto económico, legal, social y ético; entre otros- no es una cuestión trivial. No podemos negar que enfrentamos un proceso que no solo concierne al sistema normativo sino que indiscutiblemente reviste un carácter interdisciplinario.

Por otro lado, cabe mencionar que si bien el Parlamento Europeo hace alusión a una "persona electrónica"; en opinión de Ercilla el término ciber-físico es el que más se acercaría a la descripción de lo que ha de ser un robot inteligente, precisando que el término "electrónico" devendría en exiguo a la hora de identificar a los entes dotados de personalidad jurídica y sosteniendo que la denominación más correcta habría de ser de persona electro-física ya que este término respondería a la configuración misma de lo que en un futuro serían los robots, a saber, dispositivos físicos con capacidades de computación, almacenamiento y comunicación para controlar e interactuar con un proceso físico, controlados o monitoreados por algoritmos computacionales e integrados en red. ${ }^{19}$

Nos encontramos en un momento que nos permite avizorar la tendiente creación de un nuevo modelo de sociedad donde la convivencia con robots de

\footnotetext{
${ }^{19}$ Ercilla, Javier, Normas de Derecho Civil y Robótica. Robots Inteligentes, Personalidad Jurídica, Responsabilidad Civil y Regulación, Pamplona, Thomson Reuters Arazandi, 2018, pp.26.
} 
características humanas delineará el futuro de la humanidad por lo que debido a la trascendencia de este tema su planteamiento y consecuente análisis no estará exento de polémica en sus inicios.

Cabe reflexionar además en torno a la afirmación de Vallverdú ${ }^{20}$ quien señala que: "la personalidad legal que se les quiere otorgar ahora a los robots no tiene nada que ver con regular sus derechos, sino que tiene una motivación económica y busca eximir a los fabricantes de responsabilidad en los actos de los robots", ${ }^{21}$ línea de pensamiento compartida también por Ramón López de Mántaras. ${ }^{22}$

\section{Sobre la viabilidad de un Derecho de los Robots}

Señala acertadamente Filipe Maia Alexandre que, si bien es indiscutible que en todas las jurisdicciones los seres humanos tienen un estatus legal distinto, incluso entre nuestras especies esta realidad no es pacífica ni universal.

Ya en el primer corpus legal de la historia, el Código de Hammurabi, el estado legal de los hombres variaba en relación a los castigos según la riqueza que poseían. En todas las jurisdicciones que permitieron la existencia de la esclavitud, las personas que se consideraban esclavas no tenían el mismo estatus legal. Varias jurisdicciones también contemplaron diferentes estados legales para los hombres en función de la raza. Como ejemplo, en los Estados Unidos el primer voto emitido por un afroamericano no se hizo hasta el 31 de marzo de 1870 y los matrimonios interraciales solo se permitieron después de 1967 con Loving v. Virginia. También es posible encontrar jurisdicciones donde a las personas se les atribuyen diferentes estatutos legales basados en el género. Según la ley saudí, todas las mujeres deben tener un guardián masculino (wali), generalmente un padre, hermano, esposo o tío (mahram). Las mujeres tienen prohibido viajar, realizar negocios oficiales o someterse a ciertos procedimientos médicos sin el permiso del tutor masculino respectivo.

En este sentido, el estado legal de personas, animales, objetos y otras realidades (como ríos y empresas) varía de jurisdicción a jurisdicción y, con el transcurso del tiempo, incluso dentro de la misma jurisdicción y con respecto a la misma realidad.

\footnotetext{
${ }^{20}$ Especialista en Filosofía de la computación y bioética en la UAB quien también suscribió la carta a Juncker precisando que su postura no es en contra al reconocimiento del derecho de los robots porque precisamente su aspiración profesional es conseguir máquinas con conciencia y emociones.

${ }^{21}$ Rius, Mayte, "¿Urge regular ya los derechos de los robots en Europa?". La Vanguardia. [Consulta: 1 de marzo de 2019]. Disponible en: https://www.lavanguardia.com/tecnologia/20180417/442631680924/derechos-robots-ue-persona-electronica-ia.htmldigital/innovacion/2017/08/29/59a458d7ca4741cf138b4622.html

${ }^{22}$ Director del Instituto de Investigación de Inteligencia artificial del CSIC (Consejo Superior de Investigación Científica)
} 
Esta observación permitiría concluir que un estatus legal separado o una personalidad jurídica no se derivan de la calidad de la persona física, sino que son el resultado de opciones legislativas que se basan en consideraciones morales, que intentan reflejar las realidades sociales en el marco legal o que simplemente se hicieron por conveniencia legal.

Por lo tanto, dado que pareciera no haber un solo principio que dicte cuándo el sistema legal debe reconocer a una entidad como persona jurídica, ni cuándo debe denegar la personalidad jurídica, y a través de la historia no se deriva ninguna orientación sobre la mencionada institución, es pertinente analizar la posibilidad de que los agentes artificialmente inteligentes tengan el derecho de ser considerados entidades jurídicas separadas, ya sea que hacerlo refleje una realidad social o una opción conveniente desde un punto de vista legal.

Pagallo precisa las formas en que los derechos comparados pueden sistematizarse en tres grandes propuestas:

- Una primera aproximación, que únicamente examina como los robots afectan a los conceptos y principios legales tradicionales, como es el caso del concepto de persona jurídica.

- Una segunda que pretende edificar una nueva disciplina jurídica con nuevos paradigmas, como sería la responsabilidad por operaciones realizadas por sistemas robóticos autónomos.

- Finalmente y en sentido contrario la propuesta que niega su especificidad y convierte los retos que plantean los robots en cuestiones que se pueden abordar siempre dentro de los parámetros de los vigentes ordenamientos jurídicos. ${ }^{23}$

Compartimos la opinión de Barrio al señalar que los marcos jurídicos forzosamente tendrán que adaptarse ya que permanecer neutrales o indiferentes con respecto a la inclusión de la robótica y la IA no será una opción y mucho menos lo será la reclasificación de la tecnología con miras a una adecuación dentro de las categorías legales existentes; más aún cuando enfrentamos el problema de que muchos conceptos fundamentales se están tornando problemáticos como marcadores de límites como sería la integridad corporal en un mundo de interfaces hombre-máquina.

\footnotetext{
${ }^{23}$ Pagallo, Ugo, The Laws of Robots: Crimes, contracts and torts, New York, Springer, 2013, pp 37.
} 
En este orden de ideas, es evidente que existen circunstancias que justifican un tratamiento unitario del tema; entre éstas se encuentra cuestiones fácticas, es decir, las múltiples aplicaciones estudiadas pertenecen a un mismo sector de la investigación y de la innovación tecnológica -la robótica- que, aunque se sirve de diversas disciplinas auxiliares, presenta características suficientemente específicas y privativas. Además, la relevancia del tema es de carácter socioeconómico, puesto que la robótica implica un mercado en expansión, considerado estratégico sobre el plano económico y de gran impacto a nivel social, cuyo crecimiento requiere ser controlado mediante una intervención regulatoria.

\section{Consideraciones finales}

Debido al potencial que ofrece la conjunción robótica e inteligencia artificial, para transformar profundamente la sociedad tal como la conocemos, un enfoque proactivo puede ser la única forma de garantizar la sostenibilidad debido a que lo único cierto es que ningún estrato se mantendrá intacto. En este sentido la cuestión de si los agentes artificialmente inteligentes -en la medida que puedan actuar de manera autónoma y entendiendo esta autonomía como la capacidad de tomar decisiones y ponerlas en práctica en el mundo exterior independientemente del control o la influencia externos y tener experiencias subjetivas- tienen el derecho de ostentar un "estatus legal" separado necesita ser precedida por diversos cuestionamientos.

Somos de la opinión, sin embargo, que la formulación de un Derecho de los Robots deviene en un tema de impostergable análisis cuyo carácter interdisciplinario deberá también abordar los efectos secundarios que estas tecnologías emergentes y disruptivas tendrán en las intervenciones regulatorias dentro los diversos entornos.

\section{Referencias}

Barrientos, Antonio, Peñin, Luis, Balaguer, Carlos y Aracil, Santoja, Fundamentos de la Robótica, Madrid, Editorial Mc Graw Hill, 2007.

Barrio, Moises, "Del derecho de internet al derecho de los robots", en Barrio, Moises (coord.), Derecho de los Robots, Madrid, Wolters Kluwer, 2018, pp. 61-86.

Calo, Ryan, "Robots and ethics", en Lin, Patrick (coord.), Robot ethics: The ethical and social implications of robotics., Cambridge, MIT Press, 2012, pp. 159-168.

--_--, "Robots as legal metaphors", Harvard Journal of law and Technology, Estados Unidos, No. 30, 2016, pp. 209-237. 
Castromil, Juan, “Amazon usará drones Prime Air para repartir sus paquetes volando”. CLIPSET. [Consulta: 2 de marzo de 2019]. Disponible en: https://clipset.20minutos.es/amazon-usara-drones-prime-air-para-repartir-sus-paquetes/

Chávez Valdivia, Ana, Hacia el quebrantamiento de paradigmas jurídicos: La robótica y la inteligencia artificial, en: XXI Congreso Iberoamericano de Asociaciones de Derecho e Informática (2017: San Luis de Potosí, México).

Darling, Kate, "Extending legal protection to social robots: The effects of anthropomorphism, empathy, and violent behavior towards robotic objects”, en Calo, Ryan (coord.), Robot Law, Reino Unido, Edward Elgar, 2012.

Ercilla, Javier, Normas de Derecho Civil y Robótica. Robots Inteligentes, Personalidad Jurídica, Responsabilidad Civil y Regulación, Pamplona, Thomson Reuters Arazandi, 2018.

García-Prieto, Juan, “¿Qué es un robot?”, en Barrio, Moisés (coord.), Derecho de los Robots, Madrid, Wolters Kluwer, 2018, pp. 25-60.

Informe con recomendaciones destinadas a la Comisión sobre normas de Derecho civil sobre robótica (2015/2103(INL)), Diario Oficial de la Unión Europea, 27 de enero de 2017.

Lin, Patrick, Abney, Keith y Bekey, George, Robot ethics: The ethical and social implications of robotics, Cambridge, Editorial MIT Press, 2011.

Maia, Filipe, The Legal Status of Artificially Intelligent Robots, Tilburg, Universidad de Tilburg, 2017.

McCarthy, John, Minsky, Marvin, Rochester, Nathaniel y Shannon, Claude, “A proposal for the Dartmouth Summer Research Project on Artificial Intelligence”, AI Magazine, No. 4, 2006, pp. 12-14.

Pagallo, Ugo, The Laws of Robots: Crimes, contracts and torts, New York, Springer, 2013. Palmerini, Erica y Bertolini, Andrea, "Liability and risk management in robotics", en Schulze, Reiner., y Staudenmayer, Dirk (coords.), Digital Revolution: Challenges for Contract Law in Practice, Baden-Baden, Nomos, 2016, pp. 225-260.

Pooler, Michael, "Los robots de Amazon revolucionan la logística”. Expansión. [Consulta: 4 de marzo de 2019]. Disponible en: http://www.expansion.com/economia-digital/innovacion/2017/08/29/59a458d7ca4741cf138b4622.html

Resolución del Parlamento Europeo, Recomendaciones destinadas a la Comisión sobre normas de Derecho civil sobre robótica (2015/2103(INL)), Diario Oficial de la Unión Europea, Unión Europea, 16 de febrero de 2017.

Rius, Mayte, “¿Urge regular ya los derechos de los robots en Europa?”. La Vanguardia. [Consulta: 1 de marzo de 2019]. Disponible en: https://www.lavanguardia.com/ tecnologia/20180417/442631680924/derechos-robots-ue-persona-electronica-ia.htmldigital/innovacion/2017/08/29/59a458d7ca4741cf138b4622.html

Singer, Peter, Wired for War: the robotics revolution and confliction in the 21st century, New York, Editorial Penguin Press, 2009. 